\title{
Patient-Specific and Generic Immobilization Devices for Prostate Radiotherapy
}

\author{
Adam D. Melancon ${ }^{1,2}$, Rajat J. Kudchadker ${ }^{1}$, Richard Amos ${ }^{1}$, Jennifer L. Johnson ${ }^{1}$, Yongbin Zhang ${ }^{1}$, \\ Zhiqian H. Yu ${ }^{1,2}$, Lifei Zhang', Lei Dong', Andrew K. Lee ${ }^{3}$ \\ ${ }^{1}$ Departments of Radiation Physics, The University of Texas M. D. Anderson Cancer Center, Houston, USA \\ ${ }^{2}$ Program in Medical Physics, The University of Texas Graduate School of Biomedical Sciences at Houston, \\ Houston, USA \\ ${ }^{3}$ Departments of Radiation Oncology, The University of Texas M. D. Anderson Cancer Center, Houston, USA \\ Email: amelanco@mdanderson.org,rkudchad@mdanderson.org, richamos@mdanderson.org, jljohnso@mdanderson.org, \\ zyu@ChristianaCare.org, lifzhang@mdanderson.org, dong.lei@scrippshealth.org, aklee@mdanderson.org
}

Received July 10, 2013; revised August 5, 2013; accepted September 3, 2013

Copyright (C) 2013 Adam D. Melancon et al. This is an open access article distributed under the Creative Commons Attribution License, which permits unrestricted use, distribution, and reproduction in any medium, provided the original work is properly cited.

\begin{abstract}
The purpose of our study was to compare interfractional bony setup variations in pelvic anatomy with two immobilization devices, the patient-specific Vac-Lok and the generic Dual Leg Positioner system (both Civco Medical Solutions, Kalona, IA), for bilateral proton radiotherapy of the prostate. Two groups of 10 patients were studied. Computed tomography (CT) was performed three times a week, yielding $233 \mathrm{CT}$ image sets for the vacuum system group and 252 for the other group. The translational shifts of the pelvic bone and prostate and rotation of the upper femurs of the femoral heads with respect to the simulation CT images were analyzed. Along the anterior-posterior and lateral axes, mean and systematic translational variations of the pelvic bone and prostate, relative to skin fiducials, were significantly lower in the Vac-Lok group (all $\mathrm{p}<0.01)$ than in the Dual Leg Positioner group. Abduction of the upper femur, the dominant rotation, had random rotational variations of $1.9^{\circ}$ and $2.0^{\circ}$ and systematic rotations of $3.1^{\circ}$ and $2.9^{\circ}$ for the vacuum and generic system groups, respectively. Femoral abduction was highly correlated with anterior prostate displacement for both femurs in both groups $(\mathrm{p}<0.01)$. We conclude that image guidance may be needed to correct systematic translation introduced during simulation CT, particularly with the generic immobilization system. High degrees of femoral rotation may introduce prostate translation and distal misalignment of lateral proton beams with the prostate.
\end{abstract}

Keywords: Femoral Head; Interfractional Variation; Pelvic Immobilization; Proton Therapy; Prostate Cancer

\section{Introduction}

Pelvic immobilization during daily patient setup is a crucial step in managing treatment uncertainty during prostate radiotherapy. Modern conformal radiotherapy requires that all treatment uncertainties are minimized to enable the use of narrow target margins and an escalated target dose. Immobilization and daily target localization have become common at many cancer centers, creating a demand for precise analysis of residual treatment uncertainty and determination of the appropriate margins to accommodate it.

Previous studies have used port film or electronic portal imaging devices to compare patient setup uncertainties between pelvic immobilization devices [1-5] with patient setup uncertainties with and without rigid immobilization [6-10]. Analysis of pelvic anatomic variation with computed tomography (CT) interfractional patient data and CT registration software provides a precise and objective measure of pelvis translation relative to external setup reference points (e.g., BBs). In addition, this type of analysis provides the opportunity to analyze the three-dimensional rotation of the upper femur during daily setup, which could be particularly important for proton therapy with a lateral beam arrangement, as is typically used to treat the prostate. In such treatments, the proton beams pass directly through the dense bones of the upper femur and femoral heads (FHs) before reaching the prostate. It is still unknown how much range uncertainty is caused by these bony variations and whether the use of a particular immobilization device can reduce it.

It has been well documented that prostate position can change during daily patient setup for radiotherapy. 
[11-13]. It is arguable that the role of immobilization devices may be diminished with daily image-guided setup. However, it is important to note that a simple couch shift (the common type of correction under image guidance) cannot correct complicated anatomical changes resulting from improper immobilization. Few studies have addressed the magnitude of $\mathrm{FH}$ and upper femur variations resulting from patient setup and the potential effect and these variations have on daily target localization of the prostate, particularly for conventional bilateral proton treatments. To fill this gap in knowledge, we retrospectively compared two immobilization devices used for radiotherapy of the prostate. The first immobilization device, the Vac-Lok (Civco Medical Solutions, Kalona, IA), is a patient-specific device that conforms tightly to the patient's body contours. The second immobilization device, the Dual Leg Positioner (also from Civco Medical Solutions), is a non-patient-specific positioning system that reduces storage needs. We specifically analyzed and compared these two systems' ability to control rotation of the upper femur around the FH and, to a lesser extent, translation of the pelvic girdle and prostate with respect to the BBs to determine if the convenience of generic patient immobilization is obtained at the cost of patient setup precision.

\section{Materials and Methods}

\subsection{Simulation, Treatment Planning, and Delivery}

Twenty patients from two institutional review boardapproved protocols at our institution participated in a retrospective study to determine the efficacy of the immobilization devices for future proton radiotherapy patients. Patients enrolled on both protocols underwent three CT scans per week ("daily CT") for tumor localization using a CT-on-rails linear accelerator system (EXaCT; Varian Oncology Systems, Palo Alto, CA) immediately before their radiation treatment [14]. The CT-onrails CT-linear accelerator combination radiotherapy system allowed for daily $\mathrm{CT}$ imaging and treatment without removing the patient from the treatment couch. Patients on both protocols received 75.6 Gy with 8-field IMRT delivered in 42 fractions, yielding an average of $24 \mathrm{im}-$ ages sets per patient.

The first 10 patients were set up with the Vac-Lok system, a patient-specific vacuum positioning system in which a radiotranslucent cushion fixes the patient's anatomy from the upper thighs to the feet (Figure 1(a)). The patients were placed in the treatment position on a partially inflated cushion. The air in the cushion was then partially evacuated until the cushion was semi-rigid, and the therapist molded it around the area to be immobilized. Once the desired shape was achieved, more air was re- moved until the cushion became rigid. The second 10 patients were immobilized with the Dual Leg Positioner system, a vinyl-covered foam immobilization device that is indexable to improve reproducibility and that can be easily cleaned and reused (Figure 1(b)).

CT scans for treatment simulation were acquired while the patients had full bladders and empty rectums. For these scans, the patients were marked with one anterior and two lateral skin marks that reflected the position of the isocenter inside the prostate and coincided with the treatment room laser lines marked on the patient. After external fiducial markers (BBs) were placed on the patients' permanent skin marks, the patients were scanned. Each CT image had a 3-mm slice thickness, a $512 \times 512$ pixel matrix size, and a 1-mm axial resolution. The CT dataset was transferred to the Pinnacle3 treatment planning system (Philips Medical Systems, Andover, MA), and one radiation oncologist contoured the prostate, seminal vesicles, bladder, rectum, and FHs, as described below.

\subsection{Anatomical Contouring and Registration}

In order to compare the changes in FH and upper femur, the left $\mathrm{FH}$, right $\mathrm{FH}$, left femur, and right femur were contoured on each treatment planning CT as reference structures. In addition, a region of interest from the pelvic bone surrounding the prostate (including the ischium, pubic arch, and pubic symphysis) was selected to represent the pelvis bony anatomy in the bony registration (Figure 2). The reference images were exported along with the daily $\mathrm{CT}$ image sets into a non-commercial CT registration software [15]. The CT-assisted targeting software is capable of both rigid and deformable registration of soft tissue and bony structures. In this study, the software automatically registered the bony structures using rigid transformations. Each patient's pelvic anatomy was registered to the corresponding pelvic anatomy on the reference CT, and the treatment couch correction (translation relative to the position indicated by registra-
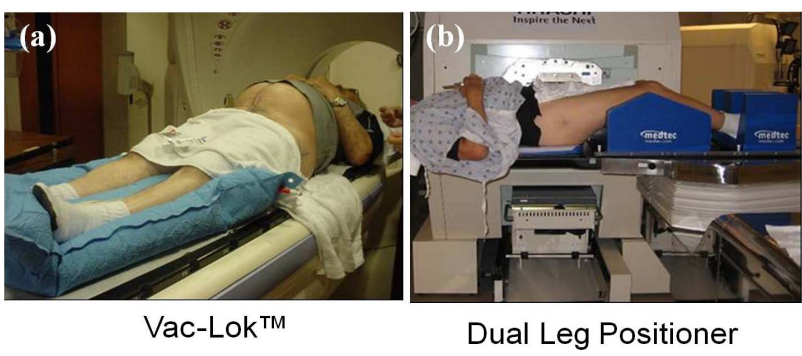

Figure 1. Two commercially available pelvic immobilization devices. The Vac-Lok system (a), a patient-specific radiotranslucent cushion that fixes the patient's anatomy from the upper thighs to the feet. The Dual Leg Positioner system (b), a non-patient-specific vinyl-covered foam immobilization device. 


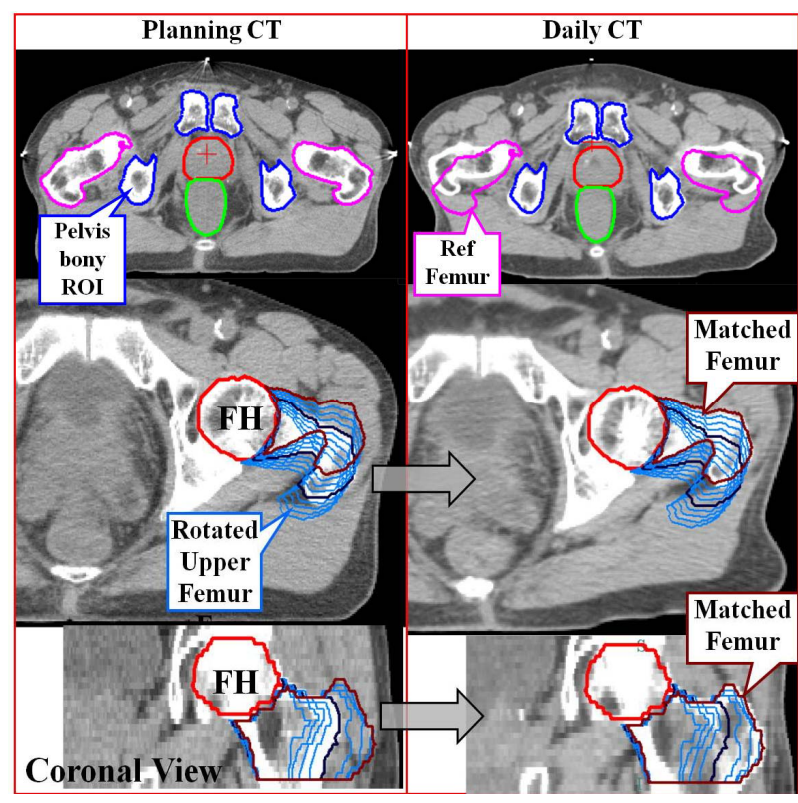

Figure 2. Pelvic bony anatomy used for CT registration (top left) and imported pelvic bone contours on daily registered pelvic bony anatomy (top right). Femoral head (FH) and upper femur contoured on the simulation CT image and contours generated from $2^{\circ}$ femoral rotations (middle left) and daily femur images matched to the rotated femurs on the planning CT image (middle right) in the axial plane. FH and upper femur contoured on the simulation CT image and contours generated from $2^{\circ}$ femoral rotations (bottom left) and daily femur images matched to the rotated femurs on the planning CT image (bottom right) in the coronal plane.

tion to the external skin fiducials) was recorded. The amount of translation of the pelvic bone relative to external skin fiducials is a good estimator of patient setup uncertainty for protocols with little or no image guidance for daily setup. Image guidance may correct translational misalignment of the target and the treatment beams.

To evaluate femoral rotation, each patient's daily FH was registered to the corresponding $\mathrm{FH}$ on the reference $\mathrm{CT}$ and the femur's contour was rotated in the axial plane around the center of the contoured $\mathrm{FH}$ volume in $2^{\circ}$ increments from the $16^{\circ}$ position to the $-16^{\circ}$ position. The contour that best matched the "daily" femur with the femur on the reference CT was manually chosen and recorded (Figure 2). This process was repeated with each daily $\mathrm{FH}$ and femur to generate the daily rotation of the left and right FHs.

\subsection{Analytical Methods}

The "daily" translations of the pelvic girdle and prostate relative to the external skin fiducials were measured first. The deviation of each patient's daily CT image set relative to the simulation $\mathrm{CT}$ image set was recorded in the superior-inferior (SI), anteroposterior (AP), and medio- lateral (or right-left $[R L]$ ) directions. The absolute mean and standard deviations were chosen to evaluate overall alignment quality between the two immobilization devices.

The daily pelvic and prostate translations were further subdivided into two components, systematic and random. The systematic component (S) and random component(s) of setup uncertainty were calculated as previously described [16,17]. S represents the patient-to-patient mean anatomical deviation from the position at simulation. $\mathrm{s}$ represents daily variation of the patient's anatomy with respect to that patient's mean position during the course of radiotherapy. Similarly, the total daily rotation of each patient's FH was recorded. The left and right FH data were combined for each group, and the mean, total uncertainty $\left(\mathrm{s}_{\text {total }}\right)$, random component of variation (s) and systematic component of variation (S) were compared.

The Student's t-test was used to analyze the statistical significance of differences in the mean and random $\mathrm{s}$ values between patient groups, for both translational and rotational variations. The F-test for equivalence of variance was used to analyze the statistical significance of differences in $\mathrm{S}$ values between the two patient groups (SPSS Inc., Chicago, IL). The Pearson's parametric correlation was use to determine correlation between rotational and translational uncertainties.

\section{Results}

There were 233 CT image sets in the vacuum system group and $252 \mathrm{CT}$ image sets in the foam immobilization system group. Table 1 shows the mean translation, s, and $\mathrm{S}$ values for daily translations of the pelvis relative to BBs in each cardinal direction. The absolute mean translation of the vacuum system group $(1.7 \mathrm{~mm})$ was statistically less than that in the other group $(2.9 \mathrm{~mm})$ along the AP axis $(p<0.01)$. The random component of uncertainty in the AP direction was greater for the vacuum system group, but the systematic component was greater in the foam system group.

The translations along the SI axis were nearly identical between the two patient groups. The generic foam immobilization system is indexed along the axis of the table and SI axis of the patient. Rigorous alignment along the SI axis when using this system appeared to provide immobilization equivalent to that afforded by the rigid vacuum immobilization system.

The absolute mean translation of the vacuum system group $(0.9 \mathrm{~mm})$ was statistically less than that in the foam immobilization system group $(2.5 \mathrm{~mm})$ along the $\mathrm{RL}$ axis $(\mathrm{p}<0.01)$. The systematic components of uncertainty in the RL direction were $1.0 \mathrm{~mm}$ for the Vac-Lok group and $3.3 \mathrm{~mm}$ for the Dual Leg Positioner group $(\mathrm{p}<0.01)$. In general, alignment to the BBs was equivalent in the SI direction and mean components of 
variations were greater along the lateral and $\mathrm{AP}$ axis for the generic immobilization device. This indicates BB alignment with the generic device may be less reproducible from the position on the reference $\mathrm{CT}$ than the vacuum device.

The translation of the prostate relative to the BBs during the same treatment sessions is summarized in Table 2. The misalignments along the SI and RL axis were nearly identical to the misalignments of the pelvic bony anatomy. However, the misalignments along the AP axis were substantially greater for the prostate than for the pelvic bony anatomy. Additional factors may have contributed to the larger uncertainty along the AP axis of the prostate, including prostate motion caused by rectal and bladder filling and deformation of the pelvic region (such as by femoral rotation).

The daily rotations of the upper femur around the FH were random about the origin (Figure 3), so the standard deviations (total uncertainty) of each group were more indicative of overall immobilization quality than the direction-specific uncertainty values. The standard deviations of the daily femoral rotations in both groups were nearly identical, $3.67^{\circ}$ for the vacuum system group and $3.57^{\circ}$ for the foam system group.

The random components of uncertainty (Table 3) were $1.9^{\circ}$ and $2.0^{\circ}$ for the vacuum and generic foam system groups, respectively. The systematic component of uncertainty was $3.1^{\circ}$ for the former group and $2.9^{\circ}$ for the latter group. There were no statistically significant differences between the two groups when daily femoral rotations were compared.

Uncertainty along the AP axis was found to be greater for the prostate than the pelvic bony anatomy. We correlated prostate misalignment with daily $\mathrm{FH}$ rotation to investigate whether rotation might have negatively impacted daily target localization of the prostate (Figure 4).

Table 1. Daily translation of pelvic bony anatomy relative to external skin marks.

\begin{tabular}{|c|c|c|c|c|c|c|c|c|c|}
\hline \multirow[b]{2}{*}{ Immobilization Device } & \multicolumn{3}{|c|}{ AP shift, $\mathrm{mm}(\mathrm{A}=+)$} & \multicolumn{3}{|c|}{ SI shift, $\mathrm{mm}(\mathrm{I}=+)$} & \multicolumn{3}{|c|}{ RL shift, $\mathrm{mm}(\mathrm{L}=+)$} \\
\hline & Mean & $\sigma$ & $\Sigma$ & Mean & $\sigma$ & $\Sigma$ & Mean & $\sigma$ & $\Sigma$ \\
\hline Vac-Lok & 1.7 & 2.6 & 2.8 & 1.3 & 1.2 & 1.5 & 0.9 & 2.7 & 1.0 \\
\hline
\end{tabular}

$\mathrm{AP}=$ anteroposterior, $\mathrm{SI}=$ superior-inferior, $\mathrm{RL}=$ mediolateral, $\sigma=$ random variation, $\Sigma=$ systematic variation.

Table 2. Daily translation of the prostate relative to skin marks.

\begin{tabular}{|c|c|c|c|c|c|c|c|c|c|}
\hline \multirow[b]{2}{*}{ Immobilization Device } & \multicolumn{3}{|c|}{ AP shift, $\mathrm{mm}(\mathrm{A}=+)$} & \multicolumn{3}{|c|}{ SI shift, $\mathrm{mm}(\mathrm{I}=+)$} & \multicolumn{3}{|c|}{ RL shift, $\mathrm{mm}(\mathrm{L}=+)$} \\
\hline & Mean & $\sigma$ & $\Sigma$ & Mean & $\sigma$ & $\Sigma$ & Mean & $\sigma$ & $\Sigma$ \\
\hline Vac-Lok & 2.6 & 3.1 & 3.6 & 1.4 & 1.9 & 1.5 & 0.9 & 2.8 & 1.0 \\
\hline Dual Leg Positioner & 3.2 & 2.8 & 4.1 & 1.6 & 1.9 & 1.5 & 2.3 & 2.9 & 3.2 \\
\hline
\end{tabular}

$\mathrm{AP}=$ anteroposterior, $\mathrm{SI}=$ superior-inferior, $\mathrm{RL}=$ mediolateral, $\sigma=$ random variation, $\Sigma=$ systematic variation.
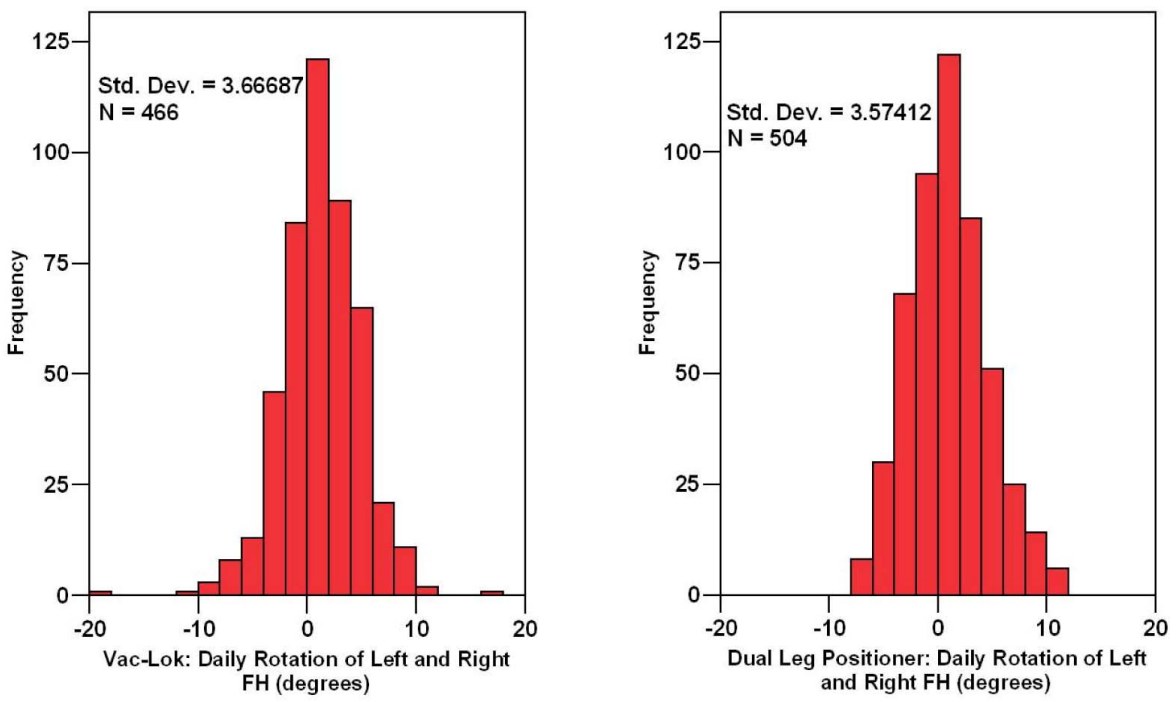

Figure 3. Total daily femoral head (FH) rotations for both groups. Histograms represent the rotations of both left and right FH combined. The total uncertainty indicated by the standard deviation suggests that the immobilization devices have nearly identical immobilization quality during treatment. 
Table 3. Total uncertainties for daily FH rotation.

\begin{tabular}{ccccc}
\hline & \multicolumn{3}{c}{ FH Rotation } \\
\hline Immobilization Device & Mean & $\sigma_{\text {total }}$ & $\sigma$ & $\Sigma$ \\
Vac-Lok & 2.4 & 3.7 & 1.9 & 3.1 \\
Dual Leg Positioner & 2.5 & 3.6 & 2.0 & 2.9 \\
\hline
\end{tabular}

$\mathrm{FH}=$ femoral head, $\sigma_{\text {total }}=$ total uncertainty, $\sigma=$ random variation, $\Sigma=$ systematic variation.

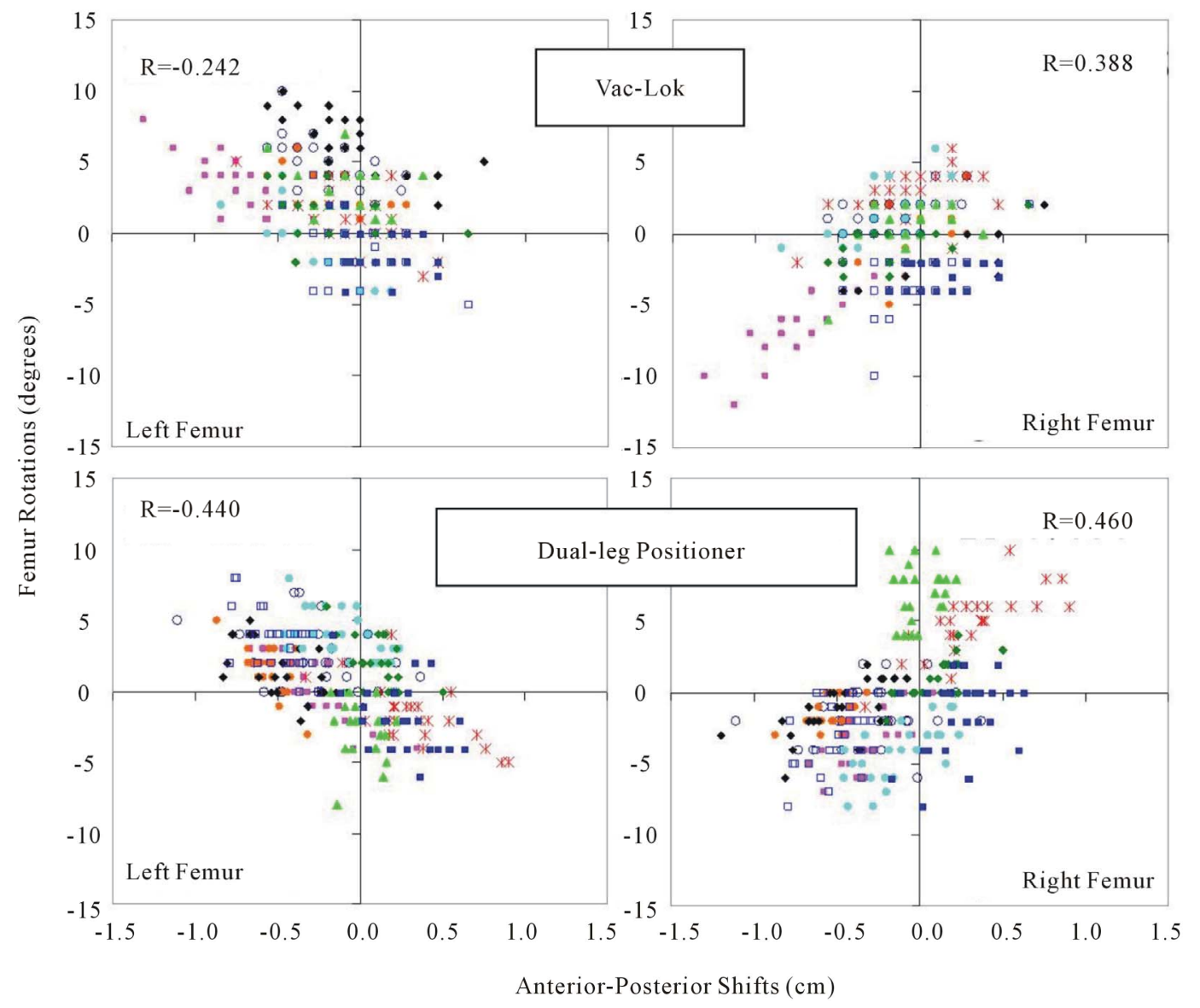

Anterior translation $(+)$. Clockwise rotation $(+)$.

Figure 4. Correlation of femoral head rotation and prostate translation in the anteroposterior direction for the Vac-Lok group (top) and Dual Leg Positioner group (bottom). A (+) indicates anterior translation or clockwise rotation. All correlations were statistically significant $(p<0.01)$. The correlation coefficients were generated with Pearson's parametric correlation. Each color corresponds to a particular patient's variations.

Right and left FH rotations for both groups were highly correlated with prostate AP interfractional motion $(\mathrm{p}<$ 0.01 , all groups), which suggests that special care to minimize FH rotation during treatment setup has the potential to improve prostate coverage during radiotherapy

\section{Discussion}

On the basis of our findings, we conclude that the VacLok pelvic immobilization device outperforms the Dual Leg Positioner particularly when considering systematic translation along the lateral axis. Treatment beam and target translational misalignments are correctable with proper image guidance and the appropriate couch shifts for correction. Additionally, for the case of lateral proton beam treatment, lateral translation of the patient does not alter the water-equivalent depth from the patient's skin surface to the distal end of the target. Thus, translational uncertainty will have minimal effects on our current proton treatment protocols.

Rotation, however, is difficult to correct. The largest component of $\mathrm{FH}$ rotation was rotation in the axial plane. Both random (s) and systematic (S) components of $\mathrm{FH}$ rotation were nearly identical between the two patient groups, at approximately $2^{\circ}$ and $3^{\circ}$, respectively. Minor 
rotations, as are seen in the majority of patients' daily treatment fractions, will have little effect on the treated volume within the pelvis. Prostate misalignment relative to the BBs was nearly equivalent to the misalignment of the pelvic bones, except along the AP axis. This additional uncertainty is due to motion of prostate within the pelvic anatomy, which is generally attributed to rectal and bladder filling [13]. However, the weak but statistically significant correlation of $\mathrm{FH}$ rotation to interfractional AP movement of the prostate suggests that femoral rotation contributes to prostate treatment uncertainty and should be managed for all radiotherapy treatments.

Femoral and FH rotations have typically been neglected in positioning studies for prostate cancer patients because their impact on patient dosimetry is minimal for photon therapy unless they result in geometric translation of the pelvis or internal pelvic organs. However, femoral and $\mathrm{FH}$ rotations may be critical to treatment uncertainties in prostate proton radiotherapy [18]. The range defined by the Bragg peak in proton therapy is roughly proportional to the radiologic path length in the beam path. Range uncertainties in the bilateral proton therapy of the prostate can potentially compromise the distal coverage of the target. In addition, dense bony structures, such as the femur and $\mathrm{FH}$, in the beam path can also cause uncertainties in dose distributions because the Bragg peak becomes degraded [19]. This degradation, in turn, affects the final dose distribution [20]. Hence, depending on the type of immobilization device used, changes in daily patient setup during the course of proton radiotherapy could introduce varying amounts of bone in the treatment field due to $\mathrm{FH}$ rotation.

It should be noted that in proton radiotherapy "smearing" $[21,22]$ is typically applied to the range compensator design in an attempt to correct for range uncertainty. Range compensators are used in proton radiotherapy to conform the distal edge of each beam to the target while accounting for the heterogeneities along each beam path. With the appropriate application of smearing in the design of each compensator, the changes in radiologic path length resulting from tissue motion should not lead to insufficient proton penetration, thus maintaining distal coverage of the target. However, compensator smearing is usually two-dimensional and orthogonal to the beam path, so significant anatomical rotation may not be fully compensated. To illustrate this insufficiency, we designed a single lateral proton beam plan with the greatest rotational misalignment found in our study (Figure 5). We found that the typical $6.6-\mathrm{mm}$ smearing in our routine proton plan for prostate treatment did not preserve the dose coverage in this case: The dose coverage for the prostate dropped from 75.6 Gy (prescribed dose) to only 60 Gy. While a single fraction of treatment with the misaligned FH may not have clinical impact, a large system-

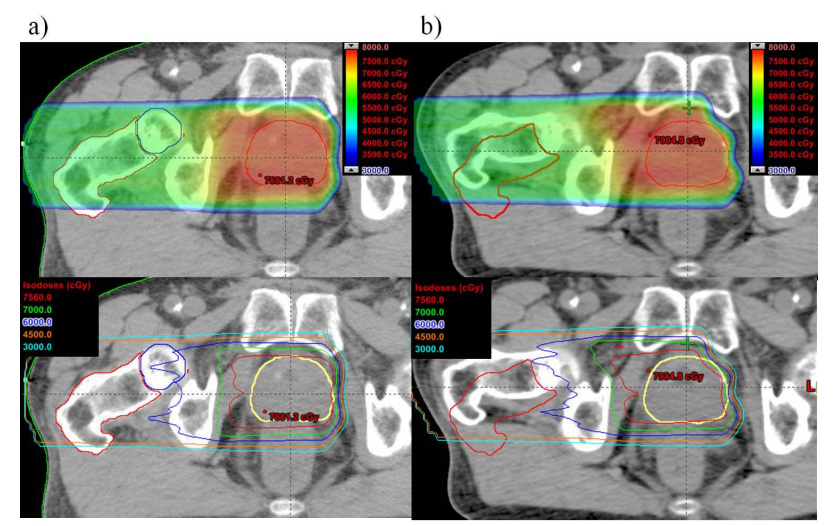

Figure 5. Changes in dose distribution to the distal edge of the prostate after a $20^{\circ}$ femoral head rotation. The reference CT image set and plan in colorwash (top) and linear (bottom) isodose lines (a). The reference plan recalculated on a daily CT image set in the Eclipse treatment planning system (Varian Medical Systems, Palo Alto, CA) (b).

atic error in $\mathrm{FH}$ position would compromise administration of the planned dose. We plan further work to evaluate the dosimetric effect resulting from patient setup uncertainties in bilateral proton therapy for prostate cancer. It is worth mentioning that the dosimetric impact of $\mathrm{FH} /$ femur position in proton therapy cannot be resolved by simply using image-guided setup techniques. Range uncertainties due to daily radiologic path length variations require the design of better immobilization devices to ensure reproducibility of the patient anatomy.

Numerous studies have been conducted on the use of immobilization for patients undergoing prostate radiotherapy. Most of these studies compared patient treatment uncertainty with and without rigid daily patient immobilization. Bentel et al. [6] compared pelvic immobilization with and without a hemibody foam cradle in 74 prostate cancer patients. They evaluated immobilization quality by comparing the number of times that the radiation oncologist requested an isocenter shift on the table. Requests were made when setup error exceeded $5 \mathrm{~mm}$. Bentel et al. found that $17.4 \%$ of patients with hemibody foam setup and $23.1 \%$ of patients without pelvic immobilization required setup correction. Soffen et al. [9] evaluated the use of rigid immobilization for the pelvic regions of early stage prostate cancer patients. The median decrease in median daily variation of patients with the immobilization devices declined to $1 / 3$ the comparison value ( $1 \mathrm{~mm}$ vs. $3 \mathrm{~mm}$ ) because of the elimination of the largest $10 \%$ of misalignments. Taken together, the results of these studies suggest that some form of daily pelvic immobilization is essential to significantly improve target reproducibility during prostate radiotherapy.

Other studies have investigated the performance of several immobilization devices and techniques. For example, in a study of 52 prostate cancer patients, Fiorino 
et al. [1] compared pelvic immobilization with an alpha cradle device fixed at the pelvic level and that with the device fixed at the leg level. The group immobilized at the leg level demonstrated smaller variations than the group immobilized at the pelvic level. In light of these findings, given the correlation between femoral rotation and prostate translation that we found in our investigation, immobilization at the leg level could have reduced femoral rotation and thus reduced prostate translation. However, in a study of 77 patients, Malone et al. [5] compared pelvic immobilization using a rubber leg cushion, a thermoplastic Hipfix (Civco Medical Solutions), and an alpha cradle device and found that the hip immobilization device was superior to the other two types. Because the results from these two studies and our investigation arrive at no consensus, we can draw no conclusions as to whether hip immobilization reduces femur rotation.

Few authors have formally addressed rotation in pelvic immobilization studies. Hanley et al. [7] measured pelvic translation and rotation in 50 prostate patients immobilized with a thermoplast body cast. They found that the pelvic rotations in the AP and SI directions were $0.6^{\circ}$ and $0.9^{\circ}$, respectively. The random and systematic components of uncertainty were respectively $1.9 \mathrm{~mm}$ and 2.0 $\mathrm{mm}$ in the RL direction, $1.4 \mathrm{~mm}$ and $1.7 \mathrm{~mm}$ in the SI direction, and $1.3 \mathrm{~mm}$ and $1.9 \mathrm{~mm}$ in the AP direction, which are in good agreement with our findings. Van Herk et al. [23] addressed femoral and prostate rotation in a study of interfractional pelvic anatomy variation with serial CT. In that study, the only significant correlations of the two presented were leg scissor with SI rotation of the prostate $(\mathrm{R}=0.47)$ and leg roll with SI rotation of the prostate $(\mathrm{R}=0.46)$. Although the magnitudes of these correlations are similar to those we reported, the magnitude, or slope, of their regression is much shallower than the regression line of prostate motion with femoral rotation in our study. The prostate would need to rotate by a large amount before geometrically shifting outside a planning target volume. Visual inspection of our correlations suggests a nearly one-to-one correlation of prostate AP shift (in $\mathrm{mm}$ ) with femoral rotation (in ${ }^{\circ}$ ). Figure 4 predicts more prostate motion for a given femoral rotation than these previous results. Thus, femoral rotation contributes more to prostate interfractional uncertainty than suggested in previous studies.

To deliver a realistic and consistent dose to patients undergoing radiotherapy, therapists must ensure that patients are adequately immobilized during simulation and treatment. Good immobilization devices should achieve true reproducibility of patient's anatomy while minimizing the additional workload introduced by other interventional, image-guided setup procedures. These devices should be relatively easy for therapists to use so that setup time is minimized and patient comfort maximized. In addition, they should be rigid and durable enough to prevent motion and last the entire course of treatment. Compared with the Vac-Lok immobilization device, the Dual Leg Positioner system displays these pivotal characteristics, making it a suitable choice for prostate radiotherapy. In addition, it can be used on multiple patients, minimizing the need for storage. Thus, we currently use this foam immobilization system in our institution for all our prostate patients undergoing IMRT and proton therapy.

\section{Conclusion}

We found that the Vac-Lok system introduced less systematic uncertainty, particularly laterally, than the Dual Leg Positioner system; however, our data suggest that these differences are minimal for institutions utilizing daily image-guided setup or treating with lateral proton beams. Rotational variations due to the two setup techniques were nearly identical. A $2^{\circ}-3^{\circ}$ random rotation (1 standard deviation) of the FH will probably not have a detrimental effect on a prostate cancer patient's proton fraction; however, a larger-than-expected systematic rotation of the $\mathrm{FH} /$ femur raises concern about the dosimetric impact of bilateral proton delivery to the prostate. Regardless of the immobilization device used or treatment modality, additional measures are needed to improve treatment precision by managing systematic $\mathrm{FH}$ rotations introduced during initial patient setup.

\section{REFERENCES}

[1] C. Fiorino, M. Reni, A. Bolognesi, A. Bonini, G. M. Cattaneo and R. Calandrino, "Set-Up Error in Supine-Positioned Patients Immobilized with Two Different Modalities during Conformal Radiotherapy of Prostate Cancer," Radiotherapy \& Oncology, Vol. 49, No. 2, 1998, pp. 133141. http://dx.doi.org/10.1016/S0167-8140(98)00127-3

[2] A. Kneebone, V. Gebski, N. Hogendoorn and S. Turner, "A Randomized Trial Evaluating Rigid Immobilization for Pelvic Irradiation," International Journal of Radiation Oncology*Biology*Physics, Vol. 56, No. 4, 2003, pp. 1105-1111.

http://dx.doi.org/10.1016/S0360-3016(03)00222-0

[3] C. Mitine, M. T. Hoornaert, A. Dutreix and M. Beauduin, "Radiotherapy of Pelvic Malignancies: Impact of Two Types of Rigid Immobilisation Devices on Localisation Errors," Radiotherapy \& Oncology, Vol. 52, No. 1, 1999, pp. 19-27. http://dx.doi.org/10.1016/S0167-8140(99)00071-7

[4] P. Y. Song, M. Washington, F. Vaida, R. Hamilton, D. Spelbring, B. Wyman, J. Harrison and G. T. Chen, "A Comparison of Four Patient Immobilization Devices in the Treatment of Prostate Cancer Patients with Three Dimensional Conformal Radiotherapy," International Journal of Radiation Oncology*Biology*Physics, Vol. 34, No. 
1, 1996, pp. 213-219. http://dx.doi.org/10.1016/0360-3016(95)02094-2

[5] S. Malone, J. Szanto, G. Perry, L. Gerig, S. Manion, S. Dahrouge and J. Crook, "A Prospective Comparison of Three Systems of Patient Immobilization for Prostate Radiotherapy," International Journal of Radiation Oncology*Biology*Physics, Vol. 48, No. 3, 2000, pp. 657-665. http://dx.doi.org/10.1016/S0360-3016(00)00682-9

[6] G. C. Bentel, L. B. Marks, G. W. Sherouse, D. P. Spencer and M. S. Anscher, "The Effectiveness of Immobilization during Prostate Irradiation," International Journal of Radiation Oncology*Biology*Physics, Vol. 31, No. 1, 1995, pp. 143-148.

http://dx.doi.org/10.1016/0360-3016(94)00351-K

[7] J. Hanley, M. A. Lumley, G. S. Mageras, J. Sun, M. J. Zelefsky, S. A. Leibel, Z. Fuks and G. J. Kutcher, "Measurement of Patient Positioning Errors in Three-Dimensional Conformal Radiotherapy of the Prostate," International Journal of Radiation Oncology*Biology*Physics, Vol. 37, No. 2, 1997, pp. 435-444.

http://dx.doi.org/10.1016/S0360-3016(96)00526-3

[8] S. A. Rosenthal, J. M. Galvin, J. W. Goldwein, A. R. Smith and P. H. Blitzer, "Improved Methods for Determination of Variability in Patient Positioning for Radiation Therapy Using Simulation and Serial Portal Film Measurements," International Journal of Radiation Oncology*Biology*Physics, Vol. 23, No. 3, 1992, pp. 621625. http://dx.doi.org/10.1016/0360-3016(92)90020-I

[9] E. M. Soffen, G. E. Hanks, C. C. Hwang and J. C. Chu, "Conformal Static Field Therapy for Low Volume Low Grade Prostate Cancer with Rigid Immobilization," International Journal of Radiation Oncology*Biology* Physics, Vol. 20, No. 1, 1991, pp. 141-146. http://dx.doi.org/10.1016/0360-3016(91)90150-3

[10] C. L. Creutzberg, V. G. Althof, M. D. de Hoog, A. G. Visser, H. Huizenga, A. Wijnmaalen and P. C. Levendag, "A Quality Control Study of the Accuracy of Patient Positioning in Irradiation of Pelvic Fields," International Journal of Radiation Oncology*Biology*Physics, Vol. 34, No. 3, 1996, pp. 697-708. http://dx.doi.org/10.1016/0360-3016(95)02034-9

[11] A. Chandra, L. Dong, E. Huang, D. A. Kuban, L. O’Neill, I. Rosen and A. Pollack, "Experience of UltrasoundBased Daily Prostate Localization," International Journal of Radiation Oncology*Biology*Physics, Vol. 56, No. 2, 2003, pp. 436-447. http://dx.doi.org/10.1016/S0360-3016(02)04612-6

[12] D. Litzenberg, L. A. Dawson, H. Sandler, M. G. Sanda, D. L. McShan, R. K. Ten Haken, K. L. Lam, K. K. Brock and J. M. Balter, "Daily Prostate Targeting Using Implanted Radiopaque Markers," International Journal of Radiation Oncology*Biology*Physics, Vol. 52, No. 3, 2002, pp. 699-703. http://dx.doi.org/10.1016/S0360-3016(01)02654-2

[13] K. M. Langen and D. T. Jones, "Organ Motion and Its Management," International Journal of Radiation Oncology*Biology*Physics, Vol. 50, No. 1, 2001, pp. 265-
278. http://dx.doi.org/10.1016/S0360-3016(01)01453-5

[14] L. Court, I. Rosen, R. Mohan and L. Dong, "Evaluation of Mechanical Precision and Alignment Uncertainties for an Integrated CT/LINAC System," Medical Physics, Vol. 30, No. 6, 2003, pp. 1198-1210.

[15] L. E. Court and L. Dong, "Automatic Registration of the Prostate for Computed-Tomography-Guided Radiotherapy," Medical Physics, Vol. 30, No. 10, 2003, pp. 27502757.

[16] H. C. de Boer, M. J. van Os, P. P. Jansen and B. J. Heijmen, "Application of the No Action Level (NAL) ProtoCol to Correct for Prostate Motion Based on Electronic Portal Imaging of Implanted Markers," International Journal of Radiation Oncology*Biology*Physics, Vol. 61, No. 4, 2005, pp. 969-983. http://dx.doi.org/10.1016/j.ijrobp.2004.09.035

[17] J. C. O'Daniel, L. Dong, L. Zhang, R. de Crevoisier, H. Wang, A. K. Lee, R. Cheung, S. L. Tucker, R. J. Kudchadker, M. D. Bonnen, J. D. Cox, R. Mohan and D. A. Kuban, "Dosimetric Comparison of Four Target Alignment Methods for Prostate Cancer Radiotherapy," International Journal of Radiation Oncology Biology Physics, Vol. 66, No. 3, 2006, pp. 883-891.

[18] S. V. Sejpal, R. A. Amos, J. B. Bluett, L. B. Levy, R. J. Kudchadker, J. Johnson, S. Choi and A. K. Lee, "Dosimetric Changes Resulting from Patient Rotational Setup Errors in Proton Therapy Prostate Plans," International Journal of Radiation Oncology*Biology*Physics, Vol. 75, No. 1, 2009, pp. 40-48.

http://dx.doi.org/10.1016/j.ijrobp.2008.08.042

[19] M. Urie, M. Goitein, W. R. Holley and G. T. Chen, "Degradation of the Bragg Peak Due to Inhomogeneities," Physics in Medicine and Biology, Vol. 31, No. 1, 1986, pp. 1-15. http://dx.doi.org/10.1088/0031-9155/31/1/001

[20] B. Schaffner, E. Pedroni and A. Lomax, "Dose Calculation Models for Proton Treatment Planning Using a Dynamic Beam Delivery System: An Attempt to Include Density Heterogeneity Effects in the Analytical Dose Calculation," Physics in Medicine and Biology, Vol. 44, No. 1, 1999, pp. 27-41. http://dx.doi.org/10.1088/0031-9155/44/1/004

[21] M. F. Moyers, D. W. Miller, D. A. Bush and J. D. Slater, "Methodologies and Tools for Proton Beam Design for Lung Tumors," International Journal of Radiation Oncology*Biology*Physics, Vol. 49, No. 5, 2001, pp. 14291438. http://dx.doi.org/10.1016/S0360-3016(00)01555-8

[22] M. Urie, M. Goitein and M. Wagner, "Compensating for Heterogeneities in Proton Radiation Therapy," Physics in Medicine and Biology, Vol. 29, No. 5, 1984, pp. 553-566. http://dx.doi.org/10.1088/0031-9155/29/5/008

[23] M. van Herk, A. Bruce, A. P. Kroes, T. Shouman, A. Touw and J. V. Lebesque, "Quantification of Organ Motion during Conformal Radiotherapy of the Prostate by Three Dimensional Image Registration," International Journal of Radiation Oncology*Biology*Physics, Vol. 33, No. 5, 1995, pp. 1311-1320.

http://dx.doi.org/10.1016/0360-3016(95)00116-6 